\title{
Angiotensin-Converting Enzyme Inhibitor and Angiotensin Receptor Blocker Use Associated with Reduced Mortality and Other Disease Outcomes in US Veterans with COVID-19
}

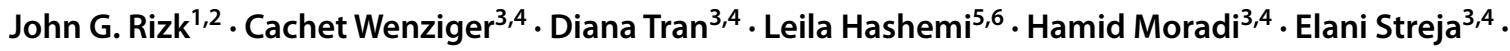 \\ Amrita Ahluwalia ${ }^{3}$ (1)
}

Accepted: 28 October 2021 / Published online: 16 December 2021

This is a U.S. government work and not under copyright protection in the U.S.; foreign copyright protection may apply 2021

\begin{abstract}
Objective To determine the association between angiotensin-converting enzyme inhibitors (ACEIs) or angiotensin II receptor blockers (ARBs) use and coronavirus disease 2019 (COVID-19) severity and outcomes in US veterans.

Patients and Methods We retrospectively examined 27,556 adult US veterans who tested positive for COVID-19 between March to November 2020. Logistic regression and Cox proportional hazards models using propensity score (PS) for weight, adjustment, and matching were used to examine the odds of an event within 60 days following a COVID-19-positive case date and time to death, respectively, according to ACEI and/or ARB prescription within 6 months prior to the COVID-19-positive case date.

Results The overlap PS weighted logistic regression model showed lower odds of an intensive care unit (ICU) admission (odds ratio [OR] 95\% CI 0.77, 0.61-0.98) and death within 60 days $(0.87,0.79-0.97)$ with an ACEI or ARB prescription. Veterans with an ARB-only prescription also had lower odds of an ICU admission (0.64, 0.44-0.92). The overlap PS weighted model similarly showed a lower risk of time to all-cause mortality in veterans with an ACEI or ARB prescription (HR [95\% CI]: 0.87, 0.79-0.97) and an ARB only prescription (0.78, 0.67-0.91). Veterans with an ACEI prescription had higher odds of experiencing a septic event within 60 days after the COVID-19-positive case date (1.22, 1.02-1.46).

Conclusion In this study of a national cohort of US veterans, we found that the use of an ACEI/ARB in patients with COVID19 was not associated with increased mortality and other worse outcomes. Future studies should examine underlying pathways and further confirm the relationship of ACEI prescription with sepsis.
\end{abstract}

Cachet Wenziger, Elani Streja, Amrita Ahluwalia have contributed equally.

Amrita Ahluwalia

amrita.ahluwalia@va.gov

$\triangle$ Elani Streja

elani.streja@va.gov

1 Arizona State University, Edson College, Phoenix, AZ, USA

2 Department of Pharmaceutical Health Services Research, University of Maryland, Baltimore, MD, USA

3 Research, Tibor Rubin VA Medical Center, VA Long Beach Healthcare System, 5901 East 7th Street, Long Beach, CA 90822, USA

4 Division of Nephrology and Hypertension, Harold Simmons Center for Kidney Disease Research and Epidemiology, School of Medicine, University of California Irvine, Orange, CA, USA

5 Greater Los Angeles VA Medical Center, Los Angeles, CA, USA

6 UCLA Geffen School of Medicine, Los Angeles, CA, USA

\section{Key Points}

ACEI/ARB use in veterans with COVID-19 was associated with reduced odds of an ICU event and mortality.

ACEI/ARB use in veterans with COVID-19 was not associated with worse outcomes such as hospitalization, acute respiratory failure, acute MI, acute kidney injury, or pneumonia.

ACEI but not ARB, use in veterans with COVID-19 was associated with increased odds of sepsis.

\section{Introduction}

The coronavirus disease 2019 (COVID-19) pandemic, caused by the severe acute respiratory syndrome coronavirus 2 (SARS-CoV-2), has resulted in an unprecedented 
worldwide public health and economic crisis [1, 2]. Angiotensin-converting enzyme inhibitors (ACEIs) and angiotensin II receptor blockers (ARBs) are widely prescribed drugs that are commonly used to manage systolic heart failure, ischemic heart disease, hypertension, and proteinuria in diabetes and chronic kidney disease (CKD). Recently, there has been much debate regarding the use of ACEIs and ARBs in patients with COVID-19, stemming from in vitro studies showing that ACEIs and ARBs upregulate the expression of ACE-2 [3-9], the receptor through which SARS-CoV-2 enters the host cells [10] in some tissues such as heart and liver and in kidney vasculature [3-8]. Based on these studies, it can be rationalized that ACEI/ARB users may have increased risk of SARS-CoV-2 infection and/or COVID-19 severity [3, 5, $11,12]$. However, recent studies in rats and humans did not confirm ACE-2 upregulation by ACEI/ARB [13-15]. Furthermore, it is not clear whether ACEI/ARB use is associated with increased risk of COVID-19. While a study of hypertension patients from an integrated healthcare system in the USA found no association between ACEI or ARB and increased likelihood of COVID-19 infection [16], another study found ACEI/ARB use to be associated with increased COVID-19 positivity or severe disease in patients from public hospitals or outpatient clinics in Hong Kong, even after adjusting for confounding variables [17].

Recent observational and clinical trials have shown conflicting data on the role of ACEI/ARB in SARSCoV-2 infection or severity and outcomes of COVID-19, but most have not shown worse outcomes [18-23]. ACE2 , the enzyme that converts angiotensin I to angiotensin II, is expressed near the surface of human epithelial cells throughout the body, including the lungs [24]. ACE-2 can also catalyze the conversion of angiotensin II to angiotensin 1-7 [25], resulting in vasodilation and anti-inflammatory, anti-oxidant, and anti-apoptotic effects [26]. ACEIs block the formation of angiotensin II, while ARBs inhibit the action of angiotensin II on its receptor [5, 27, 28].

There is no strong class 1 level evidence supporting the continuation or discontinuation of these medications, although multiple professional societies including the American Heart Association (AHA), American College of Cardiology (ACC), Heart Failure Society of America (HFSA), and European Society of Cardiology (ESC) have supported the continuation of these agents in patients who are already placed on these agents [29]. Similarly, the NIH COVID-19 Treatment Guidelines Panel states that patients on an ACEI or ARB for cardiovascular disease (or other non-COVID-19 indications) should continue these drugs during acute management of COVID-19 unless clinically indicated (e.g. hemodynamic instability) [30]. This decision is based on smaller studies or in studies of subpopulation cohorts.
Associations between ACEI/ARB and clinical outcomes in COVID-19 patients have not been examined in a large cohort with diverse demographic backgrounds and comorbidities. In this study, using a large cohort of veterans in the Department of Veterans Affairs (VA) COVID-19 Shared Data Resource, who ubiquitously use ACEI/ARBs as a part of their disease management, we sought to examine the effects of ACEI and ARB on COVID-19 severity and outcome.

\section{Methods}

This study was reviewed by the VA Long Beach Healthcare System's Institutional Review Boards (IRB) and deemed exempt from the written consent requirement since all analyses were conducted using preexisting, de-identified data. This work was supported using resources and facilities of the Department of Veterans Affairs (VA) Informatics and Computing Infrastructure (VINCI), VA HSR RES 13-457. This investigation conforms to the principles outlined in the declaration of Helsinki.

\subsection{Study Population and Data Source}

We retrospectively examined a cohort consisting of 27,556 adult veterans, aged $\geq 18$ years, with a confirmed positive test result for COVID-19 between March 1st, and November 3rd, 2020. A SARS-CoV-2 quantitative RTPCR test, performed uniformly across all VA facilities, was used to determine the positive COVID-19 test result. The date of index was the date that the SARS-CoV-2 RTPCR result was positive. Veterans were stratified based on ACEI or ARB medication use within 6 months prior to their COVID-19-positive case date. Information on ACEI and ARB medication use was obtained using VA class codes, drug description, and brand names from the VA pharmacy data sources. A total of 7207 patients were found to have an ACEI or ARB prescription (2678 patients on ACE exclusively and 4473 patients on ARB exclusively, and 56 on both) and 20,349 veterans did not have an ACEI or ARB prescription within 6 months prior to their case date. This latter unexposed group was used as the reference for all analyses. Information on body mass index (BMI), recorded within 2 years prior to the index date, outcomes within 60 days of a positive COVID-19 case date, and medications prescribed after the COVID-19-positive case date were obtained from the VA COVID-19 Shared Data Resource. Information on date of death and censoring up until November 30th, 2020, was obtained from the VA mini vital status dataset for time to death models. 


\subsection{Statistical Methods}

Baseline characteristics were stratified according to ACEI or ARB use. Characteristics were reported as mean \pm standard deviation (SD) for continuous variables or proportions $N(\%)$ for categorical variables. The two groups were compared using either a $t$-test or chi-squared test as appropriate for variable type and distribution.

A logistic regression model was used to examine the relationship between ACEI or ARB, ACEI only, and ARB only use versus no ACEI/ARB use with the outcomes of mechanical ventilation, hospitalization, intensive care unit admission (ICU), acute respiratory failure, acute myocardial infarction (MI), acute kidney injury (AKI), pneumonia, sepsis, and death within 60 days following the COVID-19-positive case date (ACEI only and ARB only analyses each excluded the 56 patients who were on both medications). A Kaplan-Meier with log-rank test and Cox proportional hazards regression model was used to examine the relationship between ACEI or ARB, ACEI only, and ARB only versus no ACE or ARB use with the outcome of time to death. Follow-up time was calculated from the COVID-19-positive case date until date of death, loss to follow-up, or the end date of the study period of November 30, 2020. Proportionality assumptions for the Cox proportional hazards regression were checked using Kaplan-Meier survival curves (Supplementary Fig. 1) and negative log-log plots. For both the logistic and Cox proportional hazards regression, an unadjusted model was created as well as an adjusted model which included the covariates of: week and VA station of the COVID-19 case date, age, sex, race, ethnicity, smoking status, comorbid MI, congestive heart failure (CHF), peripheral arterial disease (PAD), cardiovascular disease, cerebrovascular disease, chronic obstructive pulmonary disease (COPD), AKI, CKD, liver disease, diabetes, hypertension, cancer, AIDS, and Charlson Comorbidity Index (CCI) [31]. Additional factors included $\mathrm{BMI}$ at the case date; medications prescribed within 2 years prior to the case date, including metformin, calcium channel blockers (CCB), statins, beta-blockers; and lastly medications prescribed after the case date, including azithromycin, remdesivir, hydroxychloroquine, and dexamethasone.

To address the possibility of bias and confounding by indication, a propensity score (PS) analysis was conducted. The conditional probability of taking an ACEI or ARB, an ACEI only, or an ARB only was estimated using a multivariate logistic regression model, which included all variables used for model adjustment as previously stated and a PS was calculated. The PS analysis included three models: the first where veterans were matched 1-to-1 on their estimated PS, the second where the PS was included as a variable in model adjustment, and the third method of overlap PS weighting where each veteran weighs equal to the probability of being in the opposite exposure group [32, 33]. Veterans were matched using a one-to-one greedy matching method with a caliper width of 0.2 SDs without replacement. Among the PS-matched cohort, standardized differences for the matched ACEI or ARB use and non-use groups were calculated for each variable to compare the success of matching. We considered a standardized difference of greater than 0.2 to be statistically significant. Three different logistic regression models and Cox proportional hazards regression models were then repeated in the PS-matched cohort, with the PS as an adjustment variable, and weighted by the overlap PS.

A subgroup analysis was also conducted in which veterans were divided into groups based on whether or not they had comorbid hypertension. From the hypertensive group, a third subgroup was created for those with hypertension but without diabetes, CKD, or congestive heart failure. Among these three subgroups, an overlap PS weighted logistic regression model was conducted comparing the odds of the various outcomes within 60 days after the case date comparing ACEI or ARB use versus no ACEI/ARB use. Effect modification of hypertension on the association of ACEI or ARB use with the odds of an event were examined in subgroup analysis and using a Wald's test for interaction.

An additional subgroup analysis was also conducted comparing Black versus non-Black veterans. The overlap PS weighted logistic regression model was repeated among these two subgroups. Effect modification of race on the relationship of ACEI or ARB use with the odds of an event were examined using a Wald's test for interaction. Information on BMI was missing in approximately $3 \%$ of veterans and was handled using imputation by mean. Information for all other variables was complete. All analyses were performed with SAS Enterprise Guide, version 7.1 (SAS Institute Inc., Cary, NC).

\section{Results}

\subsection{Demographics and Comorbidities}

Of the 27,556 COVID-19-positive veterans, the mean \pm SD age of all veterans was $63 \pm 16$ years, $12 \%$ were female, $62 \%$ were White, $26 \%$ Black, and $10 \%$ Hispanic. Among all COVID-19-positive veterans, the comorbidities with the highest prevalence were hypertension $(62 \%)$, diabetes $(37 \%)$, and cardiovascular disease $(33 \%)$. Of the 20,349 veterans without an ACEI or ARB prescription, the mean \pm SD age was $61 \pm 18$ years, $14 \%$ were female, $61 \%$ were White, 25\% Black, and 10\% Hispanic, 51\% hypertensive, $28 \%$ diabetic, and $28 \%$ had cardiovascular disease. Compared to veterans without an ACEI/ARB, those with an ACEI/ARB were older, less likely to be female or Hispanic, more likely to be White or Black, and more 
Table 1 Baseline characteristics of COVID-19-positive veterans with an ACEI or ARB prescription 6 months prior to their positive case date compared to veterans with no ACE or ARB prescriptions between March 1st, 2020, and November 3, 2020

\begin{tabular}{|c|c|c|c|c|}
\hline \multirow[t]{2}{*}{ Variables } & \multirow{2}{*}{$\begin{array}{l}\text { Confirmed positive } \\
\text { cases } \\
N=27,556\end{array}$} & \multirow{2}{*}{$\begin{array}{l}\text { No ACEI/ARB } \\
N=20,349\end{array}$} & \multirow{2}{*}{$\begin{array}{l}\text { ACEI/ARB } \\
N=7207\end{array}$} & \multirow[t]{2}{*}{$\begin{array}{l}\text { Standardizec } \\
\text { difference }\end{array}$} \\
\hline & & & & \\
\hline Age $($ mean $\pm S D)$ & $63 \pm 16$ & $61 \pm 18$ & $68 \pm 12$ & 0.4348 \\
\hline Female $N(\%)$ & $3340(12)$ & 2947 (14) & $393(5)$ & -0.3049 \\
\hline \multicolumn{5}{|l|}{ Race $N(\%)$} \\
\hline White & $17082(62)$ & $12462(61)$ & $4620(64)$ & 0.0592 \\
\hline Black or African American & $7032(26)$ & $5047(25)$ & $1985(28)$ & 0.0624 \\
\hline Other & 3404 (12) & $2807(14)$ & $597(8)$ & -0.1765 \\
\hline \multicolumn{5}{|l|}{ Ethnicity $N(\%)$} \\
\hline Hispanic & $2728(10)$ & $2096(10)$ & $632(9)$ & -0.0521 \\
\hline \multicolumn{5}{|l|}{ Smoking $N(\%)$} \\
\hline Current & $3017(11)$ & $2276(11)$ & $741(10)$ & -0.0292 \\
\hline Past & $11180(41)$ & $7708(38)$ & $3472(48)$ & 0.2091 \\
\hline Never & $10414(38)$ & $7627(37)$ & $2787(39)$ & 0.0245 \\
\hline \multicolumn{5}{|l|}{ Comorbidities ( 2 years prior to case date) $N(\%)$} \\
\hline Myocardial infarction & $567(2)$ & $315(2)$ & $252(4)$ & 0.1245 \\
\hline Congestive heart failure & $2128(8)$ & $1263(6)$ & $865(12)$ & 0.2025 \\
\hline Peripheral arterial disease & $3101(11)$ & $1866(9)$ & $1235(17)$ & 0.2374 \\
\hline Cardiovascular disease & $9120(33)$ & $5696(28)$ & $3424(48)$ & 0.4110 \\
\hline Cerebrovascular disease & $653(2)$ & $432(2)$ & $221(3)$ & 0.0594 \\
\hline Chronic obstructive pulmonary disease & $4356(16)$ & $2968(15)$ & $1388(19)$ & 0.1249 \\
\hline Acute kidney injury & $2045(7)$ & $1323(7)$ & $722(10)$ & 0.1280 \\
\hline Chronic kidney disease & $4024(15)$ & $2494(12)$ & $1530(21)$ & 0.2421 \\
\hline Chronic kidney failure & $521(2)$ & $383(2)$ & $138(2)$ & 0.0024 \\
\hline Liver disease & $1471(5)$ & $945(5)$ & $526(7)$ & 0.1122 \\
\hline Diabetes & $10158(37)$ & $5758(28)$ & $4400(61)$ & 0.6978 \\
\hline Cancer & $4480(16)$ & $2964(15)$ & $1516(21)$ & 0.1697 \\
\hline AIDS & $194(1)$ & $163(1)$ & $31(0.4)$ & -0.0474 \\
\hline Hypertension & $17188(62)$ & $10335(51)$ & $6853(95)$ & 1.1503 \\
\hline Charlson Comorbidity Index, Median (IQR) & $2(0,3)$ & $1(0,2)$ & $2(1,4)$ & 0.4846 \\
\hline Body mass index (at case date) $($ mean $\pm \mathrm{SD})$ & $31 \pm 6$ & $30 \pm 6$ & $32 \pm 7$ & 0.2395 \\
\hline \multicolumn{5}{|l|}{ Medications ( 2 years prior to case date) $N(\%)$} \\
\hline Metformin & $5715(21)$ & $2730(13)$ & $2985(41)$ & 0.6611 \\
\hline Calcium channel blockers & $6777(25)$ & $3726(18)$ & $3051(42)$ & 0.5415 \\
\hline Statins & $13267(48)$ & $7651(38)$ & $5616(78)$ & 0.8943 \\
\hline Beta-blockers & $8434(31)$ & $4770(23)$ & $3664(51)$ & 0.5913 \\
\hline Anticoagulants & $5626(20)$ & 3414 (17) & $2212(31)$ & 0.3315 \\
\hline \multicolumn{5}{|l|}{ Medications (after case date) $N(\%)$} \\
\hline Azithromycin & $1007(4)$ & $701(3)$ & $306(4)$ & 0.0417 \\
\hline Remdesivir & $369(1)$ & $244(1)$ & $125(2)$ & 0.0445 \\
\hline Hydroxychloroquine & $278(1)$ & $206(1)$ & $72(1)$ & -0.0013 \\
\hline Dexamethasone & $639(2)$ & $404(2)$ & $235(3)$ & 0.0799 \\
\hline
\end{tabular}

$I Q R$ interquartile range, $S D$ standard deviation 
likely to have nearly all comorbidities with the exception of AIDS (Table 1). Baseline characteristics in the one-toone PS-matched cohort are summarized in Supplementary Table 1.

\subsection{Odds of Outcomes 60 Days Post-COVID-19- Positive Case Date For ACEI/ARB Use versus Non-Use}

In the unadjusted logistic regression model, veterans with ACEI or ARB use had higher odds of having an event for mechanical ventilation, hospitalization, acute respiratory failure, acute MI, AKI, pneumonia, sepsis, and death within 60 days after testing positive for COVID19 (Table 2). However, associations were attenuated to the null for all outcomes with the exception of death in models accounting for covariates (adjusted, PS adjusted, PS matched and overlap PS weighted). The overlap PS model showed ACEI or ARB use had 13\% lower odds of death within 60 days of COVID-19 case date (OR [95\% CI $0.87,0.79-0.97], p=0.0131$ ). In PS adjusted, PS matched, and the overlap PS weighted model, ACEI or ARB use was also associated with lower odds of being in the ICU within 60 days of the COVID-19 case date (OR [95\% CI $0.77,0.61-0.98, p=0.0309$ ] for the overlap PS weighted model).

When comparing ACEI only versus no ACEI use, in the overlap PS weighted model, there was a significantly higher odds of having a septic event 60 days following the COVID19-positive case date for ACEI users (OR [95\% CI 1.22, 1.02-1.46], $p=0.0283$ ) and results were null for all other outcomes (Table 3 ).

When comparing ARB only versus no ARB use, in the overlap PS model, results showed a lower odds of ICU event or death within 60 days following the COVID-19 case date for ARB users versus non-users (OR [95\% CI 0.64, 0.44-0.92], $p=0.0153$, and 0.78, 0.67-0.91, $p=0.0022$ for ICU event, and death, respectively). ICU was also significant in the PS one-to-one matched analysis (Table 4).

From the 27,556 COVID-19-positive veterans, 17,188 $(62 \%)$ were diagnosed with hypertension, from which $10,335(60 \%)$ did not have an ACEI or ARB prescription and $6853(40 \%)$ did have an ACEI or ARB prescription within 6 months prior to their case date. Therefore, a subgroup analysis was conducted comparing ACEI or ARB use versus non-use among those with comorbid hypertension and those with no hypertension (Supplementary Table 2). From the hypertensive group, an additional subgroup was analyzed looking at those who were hypertensive without diabetes, heart failure, or CKD. The overlap PS weighted logistic regression model showed that hypertensive veterans with ACEI or ARB use had lower odds of having an ICU visit or death within 60 days after their case date (OR [95\%

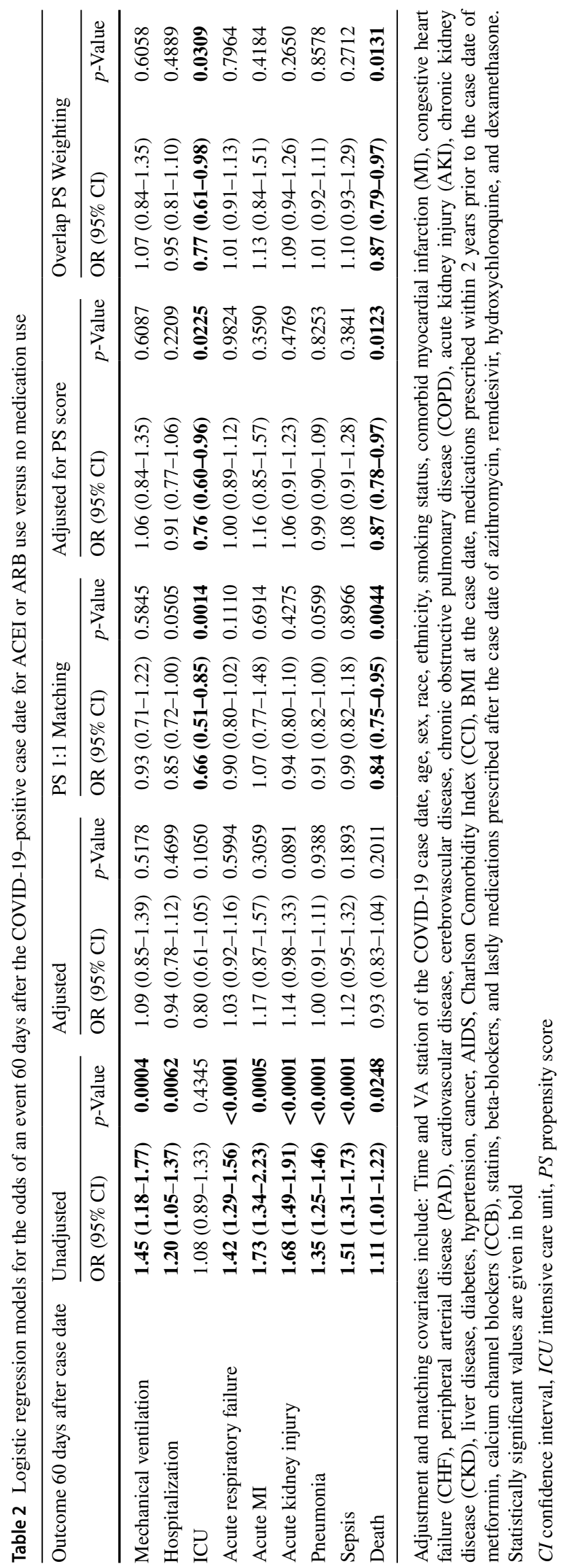




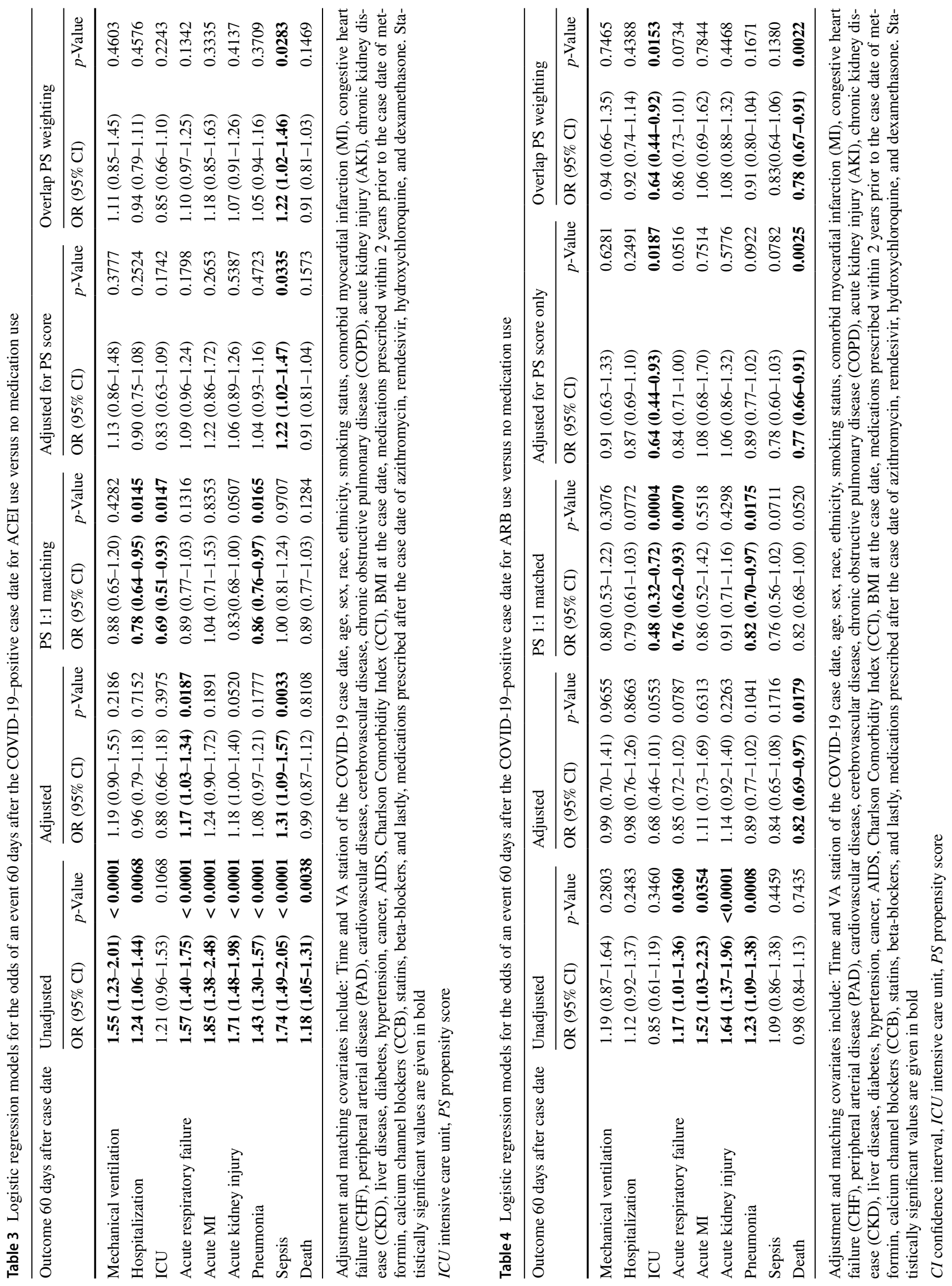


CI 0.70, 0.55-0.89], $p=0.0041$, and 0.82, 0.74-0.92, $p=$ 0.0005 for ICU, and death, respectively) (Supplementary Table 2). In contrast, non-hypertensive veterans with ACEI or ARB use had higher odds of having a hospitalization, ICU visit, acute respiratory failure, AKI, pneumonia, sepsis, or death event 60 days after their COVID-19-positive case date. For those taking ACEI or ARB medications with hypertension but without diabetes or heart failure, results were not significant for any outcome. Wald's tests for interaction between the exposure groups and hypertension were significant for the outcomes of hospitalization, ICU visit, acute respiratory failure, AKI, pneumonia, sepsis, and death within 60 days indicating that the effects of ACEI or ARB use on the odds of these outcomes varies between those with hypertension versus those without (Supplementary Table 2).

An additional subgroup analysis was conducted comparing ACEI or ARB use among Black and non-Black veterans. Among non-Black veterans, the overlap PS weighted logistic regression model showed lower odds of death within 60 days following the COVID-19-positive case date (OR [95\% CI $0.82,0.72-0.92], p=0.0012$ ) (Supplementary Table 3). For Black veterans, all associations were null. A Wald's tests for interaction between ACEI or ARB use and Black versus non-Black groups was significant for death within 60 days of the positive case date ( $p=0.0276)$ (Supplementary Table 3).

\subsection{Mortality Risk Post-COVID-19-positive Case Date for ACEI or ARB Use versus Non-Use}

Kaplan-Meier (Supplementary Fig. 1) and log-rank analysis using the overlap PS weights showed a significant difference between exposure groups $(p=0.0027)$. In Cox proportional hazards models examining time to death, the unadjusted model showed a null association between ACEI/ ARB use and risk of all-cause mortality, which remained consistent after adjusting for covariates. After one-to-one PS matching, those with ACEI or ARB use had a lower risk of mortality following COVID-19 diagnosis (HR [95\% CI $0.82(0.74-0.91], p=0.0002)$, which remained significant after adjusting for the PS (HR [95\% CI 0.87, 0.79-0.96], $p=$ 0.0075 ) and after overlap PS weighting (HR [95\% CI 0.87, $0.79-0.96], p=0.0051)$. When comparing time to death among ACEI users versus non-users, a significant reduction in mortality risk was seen only in the PS one-to-one matched model (HR [95\% CI 0.83, 0.73-0.94], $p=0.0031$ ). A significant reduction in mortality risk was seen in ARB users versus non-users in the adjusted model, the PS one-to-one matched model, the model with covariate adjustment for the PS, and also in the overlap PS weighted model (HR [95\% CI 0.78, 0.68-0.91], $p=0.001$ ) (Table 5).

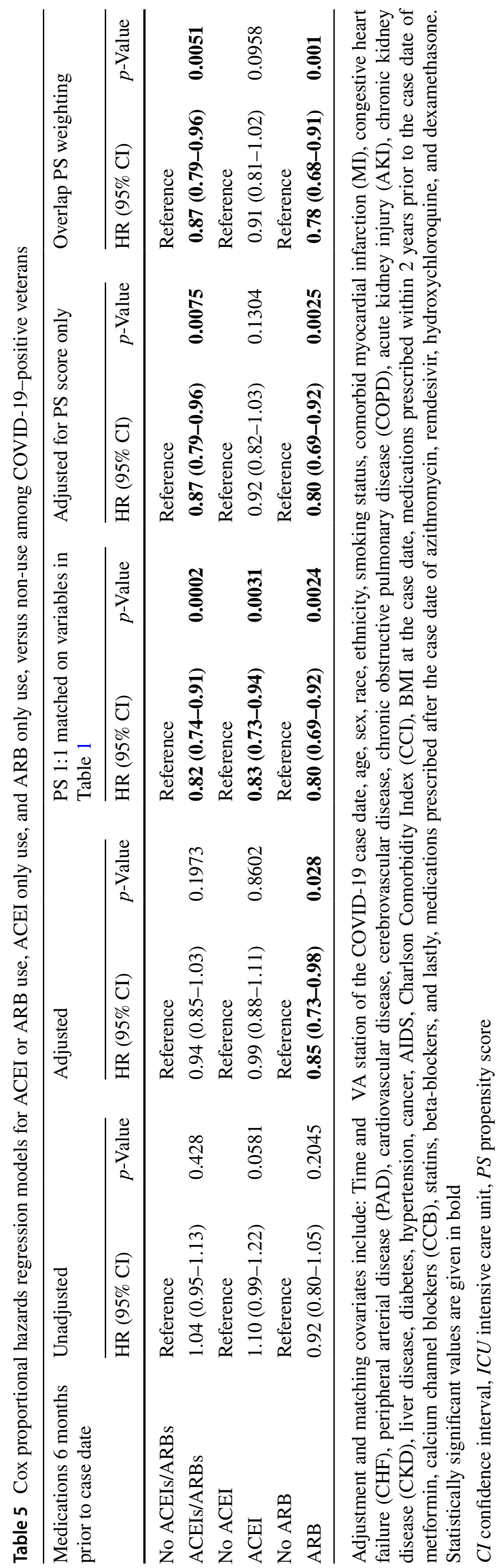




\section{Discussion}

Despite numerous recent retrospective studies that examined the association of ACEI/ARB therapy on outcomes of patients with SARS-CoV2 infection [1, 20, 34-39], the use of these pharmacologic agents in patients at risk for SARS-CoV-2 complications remains controversial. Our study in 27,556 veterans found that ACEI/ARB therapy was not associated with higher odds of most adverse outcomes in patients with COVID-19; in fact, our PS-matched model showed a reduced risk of mortality with ACEI/ARB use. However, the use of an ACEI was associated with an increased odds of sepsis amongst COVID-19 patients.

Our findings are consistent with previous reports, which have also noted reduced risk of complications and mortality in patients with COVID-19 who are being treated with ACEI/ARB [20-23, 40-43]. A multicenter study in 1128 adult patients with hypertension diagnosed with COVID19 found that the use of ACEIs/ARBs is associated with a lower risk of all-cause mortality when compared with non-users [20]. Some meta-analyses found that ACEI/ARB use is associated with reduced severity of COVID-19 disease and mortality [21-23, 40]. For instance, a randomeffects meta-analysis of 26 studies involving 8104 ACEI/ ARB users and 8203 patients who did not use ACEIs/ ARBs showed that ACEI/ARB treatment was associated with a significantly lower risk of mortality in hypertensive COVID-19 patients [41].

While the above studies demonstrated a reduction in mortality in COVID-19 patients with ACEI/ARB use, other studies found no association between these variables $[35,37,44,45]$. Khera et al showed that ACEI/ARB use was not associated with increased risk of hospitalization or inpatient mortality in a PS-matched outpatient cohort of 2263 patients with a history of hypertension as well as a positive SARS-CoV-2 test, and an inpatient cohort of 7933 hospitalized COVID-19 patients [44]. In addition, a single-center case study of 1178 COVID-19 hospitalized patients in Wuhan, China suggested that ACEI/ARB use in 115 of the 362 patients with hypertension was not associated with COVID-19 severity or mortality [37]. The discrepancy between our findings and these reports may be due to their relatively small sample size and the small number of patients using ACEIs/ARBs. Our large study cohort of veterans included $\sim 60 \%$ patients with hypertension and 26\% ACEI/ARB users. This study showed in the overlap PS weighted logistic regression model that ACEI/ ARB use reduced the odds of an ICU event and mortality.

Our study cohort comprised $26 \%$ Black patients who have a higher incidence of COVID-19 and related mortality rate when compared to other ethnic groups [46, 47]. We found that ACEI/ARB use reduced the mortality rate

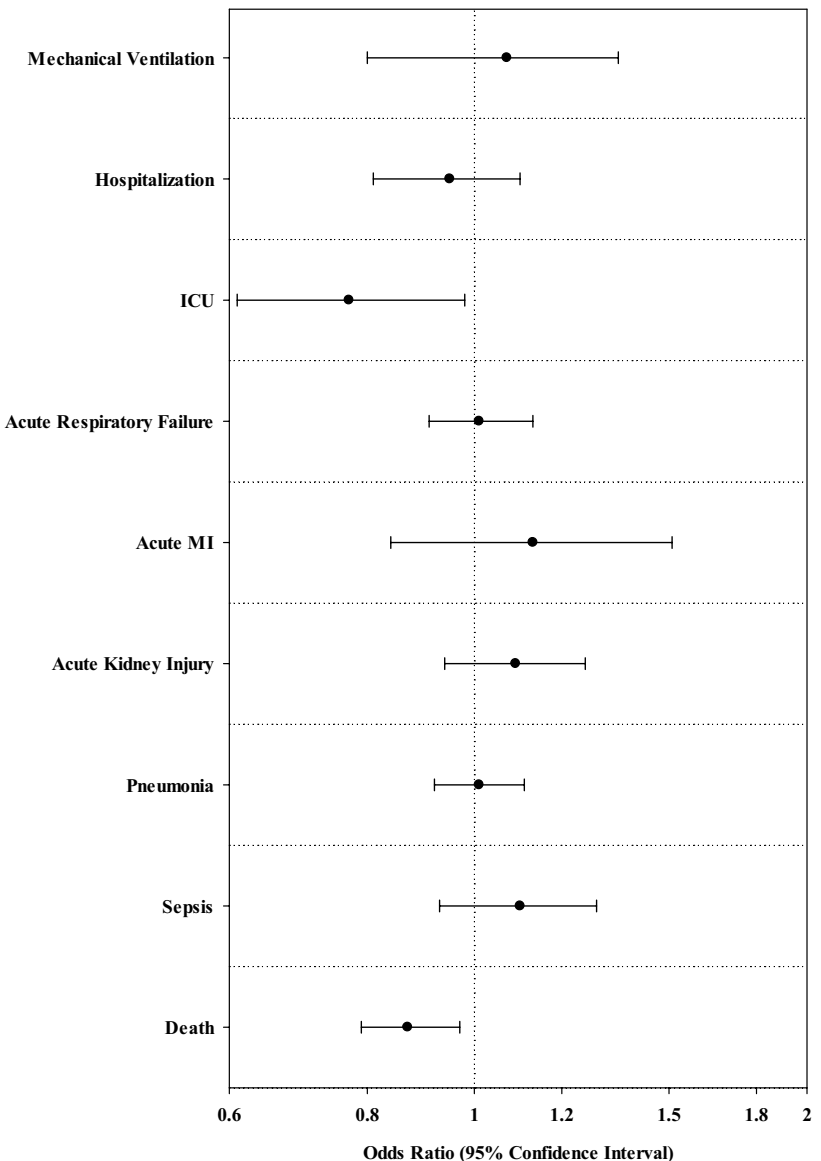

Fig. 1 Overlap propensity score weighted logistic regression model showing the odds ratio (95\% confidence interval) of an event 60 days after the COVID-19-positive case date for ACEI or ARB use. ACEI angiotensin-converting enzyme inhibitor, $A R B$ angiotensin receptor blocker, $I C U$ intensive care unit, $M I$ myocardial infarction

in non-Black veterans with COVID-19 but not in their Black counterparts. Blacks are at increased risk of hypertension, cardiovascular disease, diabetes, kidney disease, and have differences in responses to therapy [48-57]. The higher severity of comorbidities and the reduced response to ACEIs/ARBs in this population can explain the differences in mortality risk with ACEI/ARB use between Black and non-Black patients. However, future research will be needed to further examine these findings.

The average age of SARS-CoV-2-positive ACEI/ARB users in our study was 68 years. Many of these patients had other co-morbidities such as hypertension (62\%), diabetes $(37 \%)$, cardiovascular disease $(33 \%)$, and chronic lung (16\%) and kidney diseases (15\%), which have been shown to increase the risk for COVID-19-related complications and mortality [46, 58-63]. However, our study shows that the ACEI/ARB use in COVID-19 patients does not adversely affect disease outcome and may in fact contribute to reduced COVID-19-related mortality in non-Blacks. These findings 
may have important direct implications for elderly patients and those with the chronic comorbidities in the general US population.

An intersting observation of our study was that ACEI use was associated with an increased odds of sepsis amongst COVID-19 patients. These findings were in agreement with a recent study of 2700 patients admitted to ICU between January 2008 and December 2015 in Sweden that found an association between ACEI/ARB use and sepsis [64]. We did not find an association between ARB use alone and sepsis. Previous studies in COPD and hypertensive patients showed that ACEI users had a higher risk of sepsis than ARB users $[65,66]$. Given that ARBs have been shown to improve inflammation, they may, therefore, reduce the risk of sepsis [67-70]. Unlike ACEIs, ARBs have a dual role in regulating inflammation (Fig. 2); ARBs inhibit the vasoconstriction and pro-inflammatory effects of angiotensin II by blocking $\mathrm{AT}_{1}$ receptor activation, and on the other hand they mediate vasodilation and anti-inflammatory effects by activation of the $\mathrm{AT}_{2}$ receptors and Mas receptors [25, 71, 72]. In the latter secnario, ARBs cause a feedback increase in angiotensin II that can either bind to $\mathrm{AT}_{2}$ receptors directly or can be converted by ACE2 into angiotensin (1-7) which can bind to the Mas receptor and mediate vasodilation and anti-inflammatory effects [25, 71, 72]. Further studies are needed to confirm and investigate the underlying mechanisms for these findings.

ACEIs/ARBs are widely used in the treatment of hypertension, cardiovascular disease, and diabetic nephropathy. Based on previous studies that demonstrated upregulation of ACE2 in the vasculature of rodents by treatment with supra-pharmacologic doses of ACEIs/ARBs [3-8], it was postulated that ACEI/ARB use may increase the risk of
COVID-19 severity in patients. However, a few more recent studies in human and animal models did not find ACE2 upregulation with ACEI/ARBs [13-15]. Ramchand et al, while investigating the role of circulating ACE2 levels and major adverse cardiovascular events in patients with coronary artery disease, found that ACEI/ARB use did not increase the circulating ACE2 levels [13]. Burrell et al examined ACE2 expression after MI in rats and showed that an ACEI 'ramipril' had no effect on cardiac ACE2 mRNA expression [14]. A recent study in a mouse model showed that the ACEI 'captopril' and the ARB 'telmisartan' do not increase ACE2 in either lung or kidney epithelia that are target sites for SARS-CoV-2 infection [15]. These studies support our findings that the use of ACEI/ARBs does not increase the risk of adverse outcomes. Furthermore, a recent study in experimental models of acute lung injury, including a model of SARS-CoV-2 infection, suggested that ARBs may mitigate COVID-19 complications by attenuating Ang II-mediated acute lung injury via blocking AT1R [73]. These are some potential mechanisms that may underlie the reduced COVID-19 mortality rate we observed in ACEI/ARB users in our analyses. In addition, statins, with their cholesterol-lowering effects, anti-inflammatory, immunomodulatory and antiviral properties may by themselves or in combination with ACEI/ARB may modulate COVID-19 mortality [74-77].

The strengths of this study include the large sample size, use of VA Observational Medical Outcomes Partnership data as well as robust findings across multiple statistical models. Previous studies examining the association of ACEI/ARB use with COVID-19 outcomes were limited by having a considerably smaller cohort size. This study had a large sample

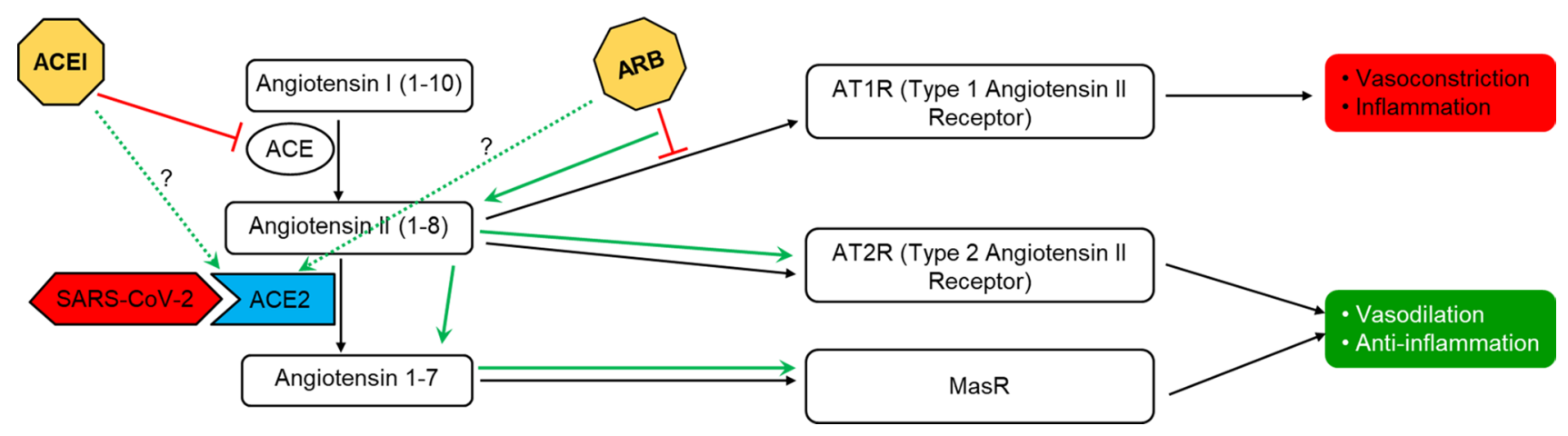

Fig. 2 SARS-CoV-2 and angiotensin-converting enzyme inhibitors (ACEIs) and angiotensin receptor blockers (ARBs). Angiotensin II is a critical regulator of blood pressure and inflammation. Upon binding to the type 1 angiotensin II receptors (AT1R) it mediates vasoconstriction and inflammation response, while upon binding to its type 2 angiotensin II receptor (AT2R) it mediates a vasodilatory and antiinflammatory response. ACEIs inhibit the conversion of angiotensin I to angiotensin II while ARBs block the binding of angiotensin II to AT1R. Some studies have suggested that these medications may increase the expression of angiotensin-converting enzyme 2 (ACE2 ), which acts as a receptor for the SARS-CoV-2 virus to infect the host cells. Physiologically, ACE-2 enzyme coverts angiotensin II to angiotensin (1-7); the latter can bind to the Mas receptor (MasR) and mediate vasodilation and an anti-inflammatory response. ARBs cause a feedback increase in angiotensin II that can either bind to AT2R receptors directly or can be converted by ACE-2 into angiotensin (1-7), which can bind to MasR, and mediate vasodilation and antiinflammatory effects 
size of 27,556 patients and included extensive adjustment for confounding factors. The VA patient population, which includes a signficant proportion of elderly patients with multiple comorbid conditions, is also suited for examining the impact of ACEI/ARB in the most vulnerable high-risk groups for COVID-19. However, the authors would also like to note several limitations of this study. Despite extensive adjustment for variables in this observational cohort study, we cannot rule out residual confounding nor make causal inferences. We were unable to adjust for other potential confounders including markers of COVID-19 disease severity such as inflammatory cytokines. Our source for exposure and outcome data were administrative electronic medical records, and there remains possible exposure or outcome misclassification. Finally, our results may not be generalizable to the general population given that our cohort consisted of VA patients who are primarily older men.

\section{Conclusion}

Our study further suggests that ACEIs and ARBs confer potent dual effects, not only in the prevention and treatment of cardiovascular disease, but also as anti-inflammatory and immunomodulatory agents [78]. Our findings are in line with the recommendations of multiple professional societies and the NIH COVID-19 Treatment Guidelines that support the continuation of ACEI and ARBs in patients who are currently prescribed such agents, considering that patients with cardiovascular disease are at increased risk of severe COVID-19 [79]. Clinical trials will also be important in examining the impact of ACEI/ARB use on clinical outcomes in COVID patients. The effect of de novo ACEI/ARB use in COVID-19 patients also merits further study.

Supplementary Information The online version contains supplementary material available at https://doi.org/10.1007/s40265-021-01639-2.

\section{Declarations}

Source of Funding This study was supported by the COVID Rapid Response award COVID-19-8900-18 from the US Veterans Administration Clinical Services Research \& Development Program (to ES, HM, and AA). The data reported here have been supplied by the US Veterans Administration. Opinions expressed in this presentation are those of the authors and do not represent the official opinion of the US Department of Veterans Affairs or the US Government. ES is supported by a career development award from the Office of Research and Development of the Department of Veterans Affairs (IK2- CX 00126601). HM is supported by a career development award from the Office of Research and Development of the Department of Veterans Affairs (1IK- CX 001043-01A2)

Ethics Approval This study was approved by the VA Long Beach Healthcare System's Institutional Review Boards (IRB) and deemed exempt from the written consent requirement since all analyses were conducted using preexisting, de-identified data. This investigation conforms to the principles outlined in the declaration of Helsinki.

Data Availability Restrictions apply to the availability of data generated or analyzed during this study. The United States Department of Veterans Affairs (VA) places legal restrictions on access to veteran's health care data, which includes both identifying data and sensitive patient information. The corresponding author will on request detail the restrictions and any conditions under which access to some data may be provided.

Disclosures HM has received research funding from Novartis and Amgen. ES has received support from Astra Zeneca and Edwards Lifesciences.

Authors' contributions Study concept and design: AA, ES, HM. Acquisition of data: CW, AA, ES, HM. Analysis and interpretation of data: CW, AA, ES, HM, JGR, LH. Statistical analysis: CW, AA, ES, HM Drafting of the manuscript: CW, AA, ES, HM, JGR, LH .Critical revision of the revised manuscript: CW, DT, AA, ES, HM, JGR, LH.

\section{References}

1. Nicola M, Alsafi Z, Sohrabi C, Kerwan A, Al-Jabir A, Iosifidis $\mathrm{C}$ et al. The socio-economic implications of the coronavirus and COVID-19 Pandemic: a review. Int J Surg. 2020;78:185-193.

2. Guo J, Huang Z, Lin L, Lv J. Coronavirus Disease 2019 (COVID19) and Cardiovascular Disease: a Viewpoint on the Potential Influence of Angiotensin-Converting Enzyme Inhibitors/Angiotensin Receptor Blockers on Onset and Severity of Severe Acute Respiratory Syndrome Coronavirus 2 Infection. J Am Heart Assoc. 2020;9:e016219.

3. Ishiyama Y, Gallagher PE, Averill DB, Tallant EA, Brosnihan KB, Ferrario CM. Upregulation of angiotensin-converting enzyme 2 after myocardial infarction by blockade of angiotensin II receptors. Hypertension. 2004;43:970-6.

4. Soler MJ, Ye M, Wysocki J, William J, Lloveras J, Batlle D. Localization of ACE2 in the renal vasculature: amplification by angiotensin II type 1 receptor blockade using telmisartan. Am J Physiol Renal Physiol. 2009;296:F398-405.

5. Ferrario CM, Jessup J, Chappell MC, Averill DB, Brosnihan KB, Tallant EA, et al. Effect of angiotensin-converting enzyme inhibition and angiotensin II receptor blockers on cardiac angiotensinconverting enzyme 2. Circulation. 2005;111:2605-10.

6. Ferrario CM, Ahmad S, Groban L. Mechanisms by which angiotensin-receptor blockers increase ACE2 levels. Nat Rev Cardiol. 2020;17:378.

7. Patel VB, Clarke N, Wang Z, Fan D, Parajuli N, Basu R, et al. Angiotensin II induced proteolytic cleavage of myocardial ACE2 is mediated by TACE/ADAM-17: a positive feedback mechanism in the RAS. J Mol Cell Cardiol. 2014;66:167-76.

8. Huang ML, Li X, Meng Y, Xiao B, Ma Q, Ying SS, et al. Upregulation of angiotensin-converting enzyme (ACE) 2 in hepatic fibrosis by ACE inhibitors. Clin Exp Pharmacol Physiol. 2010;37:e1-6.

9. Pinto BGG, Oliveira AER, Singh Y, Jimenez L, Goncalves ANA, Ogava RLT, et al. ACE2 expression is increased in the lungs of patients with comorbidities associated with severe COVID-19. J Infect Dis. 2020;222:556-63.

10. Hoffmann M, Kleine-Weber H, Schroeder S, Krüger N, Herrler T, Erichsen S, et al. SARS-CoV-2 cell entry depends on ACE2 and TMPRSS 2 and is blocked by a clinically proven protease inhibitor. Cell. 2020;181:271-280.e278. 
11. Karram T, Abbasi A, Keidar S, Golomb E, Hochberg I, Winaver J, et al. Effects of spironolactone and eprosartan on cardiac remodeling and angiotensin-converting enzyme isoforms in rats with experimental heart failure. Am J Physiol Heart Circ Physiol. 2005;289:H1351-1358.

12. Ye R, Liu Z. ACE2 exhibits protective effects against LPS-induced acute lung injury in mice by inhibiting the LPS-TLR4 pathway. Exp Mol Pathol. 2020;113:104350.

13. Ramchand J, Patel SK, Srivastava PM, Farouque O, Burrell LM. Elevated plasma angiotensin converting enzyme 2 activity is an independent predictor of major adverse cardiac events in patients with obstructive coronary artery disease. PLoS ONE. 2018;13:e0198144.

14. Burrell LM, Risvanis J, Kubota E, Dean RG, MacDonald PS, Lu $\mathrm{S}$, et al. Myocardial infarction increases ACE2 expression in rat and humans. Eur Heart J. 2005;26:369-75 (discussion 322-364).

15. Wysocki J, Lores E, Ye M, Soler MJ, Batlle D. Kidney and lung ACE2 expression after an ACE inhibitor or an Ang II receptor blocker: implications for COVID-19. J Am Soc Nephrol. 2020;31:1941-3.

16. An J, Wei R, Zhou H, Luong TQ, Gould MK, Mefford MT et al. Angiotensin-converting enzyme inhibitors or angiotensin receptor blockers use and COVID-19 infection among 824650 patients with hypertension from a US integrated healthcare system. J Am Heart Assoc 2020;10:e19669.

17. Tse G, Zhou J, Lee S, Wong WT, Li X, Liu T, et al. Relationship between angiotensin-converting enzyme inhibitors or angiotensin receptor blockers and COVID-19 incidence or severe disease. J Hypertens. 2021;39:1717-24.

18. Cohen JB, Hanff TC, Corrales-Medina V, William P, Renna N, Rosado-Santander NR, et al. Randomized elimination and prolongation of ACE inhibitors and ARBs in coronavirus 2019 (REPLACE COVID) Trial Protocol. J Clin Hypertens (Greenwich). 2020;22:1780-8.

19. Lopes RD, Macedo AVS, de Barros ESPGM, Moll-Bernardes RJ, Dos Santos TM, Mazza L, et al. Effect of discontinuing vs continuing angiotensin-converting enzyme inhibitors and angiotensin II receptor blockers on days alive and out of the hospital in patients admitted with COVID-19: a randomized clinical trial. JAMA. 2021;325:254-64.

20. Zhang P, Zhu L, Cai J, Lei F, Qin JJ, Xie J et al. Association of inpatient use of angiotensin converting enzyme inhibitors and angiotensin II receptor blockers with mortality among patients with hypertension hospitalized with COVID-19. Circ Res. 2020;126:1671-1681.

21. Baral R, White M, Vassiliou VS. Effect of Renin-AngiotensinAldosterone System Inhibitors in Patients with COVID-19: a Systematic Review and Meta-analysis of 28,872 Patients. Curr Atheroscler Rep. 2020;22:61.

22. Barochiner J, Martínez R. Use of inhibitors of the renin-angiotensin system in hypertensive patients and COVID-19 severity: a systematic review and meta-analysis. J Clin Pharm Ther. 2020;45:1244-52.

23. Liu X, Long C, Xiong Q, Chen C, Ma J, Su Y et al. Association of angiotensin converting enzyme inhibitors and angiotensin II receptor blockers with risk of COVID-19, inflammation level, severity, and death in patients with COVID-19: A rapid systematic review and meta-analysis. Clin Cardiol. 2020. https://doi.org/10. 1002/clc.23421

24. Lu R, Zhao X, Li J, Niu P, Yang B, Wu H, et al. Genomic characterisation and epidemiology of 2019 novel coronavirus: implications for virus origins and receptor binding. Lancet. 2020;395:565-74.

25. Santos RAS, Sampaio WO, Alzamora AC, Motta-Santos D, Alenina N, Bader M, et al. The ACE2/Angiotensin-(1-7)/MAS
Axis of the Renin-Angiotensin System: Focus on Angiotensin-(1-7). Physiol Rev. 2018;98:505-53.

26. Tikellis C, Bernardi S, Burns WC. Angiotensin-converting enzyme 2 is a key modulator of the renin-angiotensin system in cardiovascular and renal disease. Curr Opin Nephrol Hypertens. 2011;20:62-8

27. Dendorfer A, Dominiak P, Schunkert H. ACE inhibitors and angiotensin II receptor antagonists. Handb Exp Pharmacol. 2005. https://doi.org/10.1007/3-540-27661-0_15.

28. Werner C, Baumhakel M, Teo KK, Schmieder R, Mann J, Unger T, et al. RAS blockade with ARB and ACE inhibitors: current perspective on rationale and patient selection. Clin Res Cardiol. 2008;97:418-31.

29. Bozkurt B, Kovacs R, Harrington B. Joint HFSA/ACC/AHA statement addresses concerns Re: using RAAS antagonists in COVID-19. J Card Fail. 2020;26:370.

30. COVID-19 Treatment Guidelines Panel. Coronavirus disease 2019 (COVID-19) treatment guidelines. Updated 2021, Apr 21, vol. 2021. National Institutes of Health.

31. Charlson ME, Pompei P, Ales KL, MacKenzie CR. A new method of classifying prognostic comorbidity in longitudinal studies: development and validation. J Chronic Dis. 1987;40:373-83.

32. Li F, Thomas LE, Li F. Re: "Addressing Extreme Propensity Scores Via the Overlap Weights." Am J Epidemiol. 2021;190:189-90.

33. Li F, Thomas LE, Li F. Addressing extreme propensity scores via the overlap weights. Am J Epidemiol. 2019;188:250-7.

34. Reynolds HR, Adhikari S, Pulgarin C, Troxel AB, Iturrate E, Johnson SB, et al. Renin-angiotensin-aldosterone system inhibitors and risk of covid-19. N Engl J Med. 2020;382:2441-8.

35. Zhang X, Yu J, Pan LY, Jiang HY. ACEI/ARB use and risk of infection or severity or mortality of COVID-19: a systematic review and meta-analysis. Pharmacol Res. 2020;158:104927.

36. Bae DJ, Tehrani DM, Rabadia SV, Frost M, Parikh RV, CalfonPress M, et al. Angiotensin converting enzyme inhibitor and angiotensin II receptor blocker use among outpatients diagnosed with COVID-19. Am J Cardiol. 2020;132:150-7.

37. Li J, Wang X, Chen J, Zhang H, Deng A. Association of ReninAngiotensin System Inhibitors With Severity or Risk of Death in Patients With Hypertension Hospitalized for Coronavirus Disease 2019 (COVID-19) Infection in Wuhan, China. JAMA Cardiol. 2020;5:825-830.

38. Hippisley-Cox J, Young D, Coupland C, Channon KM, Tan PS, Harrison DA, et al. Risk of severe COVID-19 disease with ACE inhibitors and angiotensin receptor blockers: cohort study including 8.3 million people. Heart. 2020;106:1503-11.

39. Mancia G, Rea F, Ludergnani M, Apolone G, Corrao G. Reninangiotensin-aldosterone system blockers and the risk of Covid-19. N Engl J Med. 2020;382:2431-40.

40. Garg A, Rout A, Sharma A, Fiorello B, Kostis JB. Association of renin-angiotensin system blockers with outcomes in patients with COVID-19. Mayo Clin Proc. 2020;95:2559-61.

41. Wang Y, Chen B, Li Y, Zhang L, Wang Y, Yang S, et al. The use of renin-angiotensin-aldosterone system (RAAS) inhibitors is associated with a lower risk of mortality in hypertensive COVID19 patients: a systematic review and meta-analysis. J Med Virol. 2021;93:1370-7.

42. Jia N, Zhang G, Sun X, Wang Y, Zhao S, Chi W et al. Influence of angiotensin converting enzyme inhibitors/angiotensin receptor blockers on the risk of all-cause mortality and other clinical outcomes in patients with confirmed COVID-19: A systemic review and meta-analysis. J Clin Hypertens (Greenwich). 2021.

43. Vahedian-Azimi A, Rahimibashar F, Najafi A, Kidde J, Shahriary A, Shojaei S, et al. Associastion of In-hospital Use of Statins, 
Aspirin, and Renin-Angiotensin-Aldosterone Inhibitors with Mortality and ICU Admission Due to COVID-19. Adv Exp Med Biol. 2021;1327:205-14.

44. Khera R, Clark C, Lu Y, Guo Y, Ren S, Truax B et al. Association of Angiotensin-Converting Enzyme Inhibitors and Angiotensin Receptor Blockers with the Risk of Hospitalization and Death in Hypertensive Patients with Coronavirus Disease-19. J Am Heart Assoc. 2021. https://doi.org/10.1101/2020.05.17.20104943.

45. Xu J, Teng Y, Shang L, Gu X, Fan G, Chen Y et al. The effect of prior ACEI/ARB treatment on COVID-19 susceptibility and outcome: a systematic review and meta-analysis. Clin Infect Dis. 2020;72(11):e901-e913.

46. Dorn AV, Cooney RE, Sabin ML. COVID-19 exacerbating inequalities in the US. Lancet. 2020;395:1243-4.

47. Laurencin CT, McClinton A. The COVID-19 pandemic: a call to action to identify and address racial and ethnic disparities. J Racial Ethn Health Disparities 2020;7:398-402.

48. Bell CN, Thorpe RJ, Bowie JV, LaVeist TA. Race disparities in cardiovascular disease risk factors within socioeconomic status strata. Ann Epidemiol. 2018;28:147-52.

49. Adams ML, Katz DL, Grandpre J. Population-Based Estimates of Chronic Conditions Affecting Risk for Complications from Coronavirus Disease, United States. Emerg Infect Dis 2020;26(8):1831-1833. https://doi.org/10.3201/eid2608.200679.

50. Carnethon MR, Pu J, Howard G, Albert MA, Anderson CAM, Bertoni AG, et al. Cardiovascular health in African Americans: A scientific statement from the american heart association. Circulation. 2017; 136:e393-423.

51. Ejike CO, Dransfield MT, Hansel NN, Putcha N, Raju S, Martinez $\mathrm{CH}$, et al. Chronic obstructive pulmonary disease in America's Black Population. Am J Respir Crit Care Med. 2019;200:423-30

52. Laster M, Shen JI, Norris KC. Kidney disease among african americans: a population perspective. Am J Kidney Dis. 2018;72:S3-7.

53. Kovesdy CP, Norris KC, Boulware LE, Lu JL, Ma JZ, Streja E, et al. Association of race with mortality and cardiovascular events in a large cohort of US Veterans. Circulation. 2015;132:1538-48.

54. Bosworth HB, Dudley T, Olsen MK, Voils CI, Powers B, Goldstein MK, et al. Racial differences in blood pressure control: potential explanatory factors. Am J Med. 2006;119(70):e79-e15.

55. Cushman WC, Reda DJ, Perry HM, Williams D, Abdellatif M, Materson BJ. Regional and racial differences in response to antihypertensive medication use in a randomized controlled trial of men with hypertension in the United States. Department of Veterans Affairs Cooperative Study Group on Antihypertensive Agents. Arch Intern Med. 2000;160:825-31.

56. Papademetriou V, Narayan P, Kokkinos P. Angiotensin-converting enzyme inhibitors and angiotensin receptor blockers in AfricanAmerican patients with hypertension. J Clin Hypertens (Greenwich). 2004;6:310-4.

57. Weir MR, Gray JM, Paster R, Saunders E. Differing mechanisms of action of angiotensin-converting enzyme inhibition in black and white hypertensive patients. The Trandolapril Multicenter Study Group. Hypertension. 1995;26:124-30.

58. Siordia JA. Epidemiology and clinical features of COVID-19: a review of current literature. J Clin Virol. 2020;127:104357.

59. Chen N, Zhou M, Dong X, Qu J, Gong F, Han Y, et al. Epidemiological and clinical characteristics of 99 cases of 2019 novel coronavirus pneumonia in Wuhan, China: a descriptive study. Lancet. 2020;395:507-13.

60. Wenham C, Smith J, Morgan R, Group GaC-W. COVID-19: the gendered impacts of the outbreak. Lancet. 2020;395:846-8.

61. Laster Pirtle WN. Racial Capitalism: A Fundamental Cause of Novel Coronavirus (COVID-19) Pandemic Inequities in the United States. Health Educ Behav 2020; p. 1090198120922942.
62. Chung RY, Dong D, Li MM. Socioeconomic gradient in health and the covid-19 outbreak. BMJ. 2020;369:m1329.

63. de Groot NG, Bontrop RE. COVID-19 pandemic: is a genderdefined dosage effect responsible for the high mortality rate among males? Immunogenetics. 2020;72:275-277.

64. Sunden-Cullberg J. Chronic use of angiotensin-converting enzyme inhibitors and angiotensin II receptor blockers is high among intensive care unit patients with non-COVID-19 sepsis but carries a moderately increased risk of death. Hypertension. 2020;75:e15-6.

65. Lai CC, Wang YH, Wang CY, Wang HC, Yu CJ, Chen L, et al. Risk of sepsis and mortality among patients with chronic obstructive pulmonary disease treated with angiotensin-converting enzyme inhibitors or angiotensin receptor blockers. Crit Care Med. 2019;47:e14-20.

66. Dial S, Nessim SJ, Kezouh A, Benisty J, Suissa S. Antihypertensive agents acting on the renin-angiotensin system and the risk of sepsis. Br J Clin Pharmacol. 2014;78:1151-8.

67. Zhong JC, Ye JY, Jin HY, Yu X, Yu HM, Zhu DL, et al. Telmisartan attenuates aortic hypertrophy in hypertensive rats by the modulation of ACE2 and profilin-1 expression. Regul Pept. 2011;166:90-7.

68. Simoes e Silva AC, Silveira KD, Ferreira AJ, Teixeira MM ACE2, angiotensin-(1-7) and Mas receptor axis in inflammation and fibrosis. Br J Pharmacol. 2013;169:477-92.

69. Clancy P, Koblar SA, Golledge J. Angiotensin receptor 1 blockade reduces secretion of inflammation associated cytokines from cultured human carotid atheroma and vascular cells in association with reduced extracellular signal regulated kinase expression and activation. Atherosclerosis. 2014;236:108-15.

70. Callera GE, Antunes TT, Correa JW, Moorman D, Gutsol A, He $\mathrm{Y}$ et al. Differential renal effects of candesartan at high and ultrahigh doses in diabetic mice-potential role of the ACE2/AT2R/ Mas axis. Biosci Rep. 2016;36(5):e00398. https://doi.org/10.1042/ BSR20160344.

71. Siragy HM. The role of the AT2 receptor in hypertension. Am J Hypertens. 2000;13:62S-67S.

72. Dandona P, Dhindsa S, Ghanim H, Chaudhuri A. Angiotensin II and inflammation: the effect of angiotensin-converting enzyme inhibition and angiotensin II receptor blockade. J Hum Hypertens. 2007;21:20-7.

73. Kuba K, Imai Y, Rao S, Gao H, Guo F, Guan B, et al. A crucial role of angiotensin converting enzyme 2 (ACE2) in SARS coronavirus-induced lung injury. Nat Med. 2005;11:875-9.

74. Fares A, Borrmann D, Ivester JR. Are statins beneficial for the treatment of SARS-CoV-2 infection? J Infect Prev. 2021;22:177-80.

75. Katsiki N, Banach M, Mikhailidis DP. Lipid-lowering therapy and renin-angiotensin-aldosterone system inhibitors in the era of the COVID-19 pandemic. Arch Med Sci. 2020;16:485-9.

76. Kow CS, Hasan SS. The association between the use of statins and clinical outcomes in patients with COVID-19: a systematic review and meta-analysis. Am J Cardiovasc Drugs. 2021. https:// doi.org/10.1007/s40256-021-00490-w.

77. Tandaju JR, Ii W, Barati-Boldaji R, Raeisi-Dehkordi H. Metaanalysis of statin and outcomes of coronavirus disease 2019 (COVID-19): reconsideration is needed. Nutr Metab Cardiovasc Dis. 2021;31:2737-9.

78. Rizk JG, Kalantar-Zadeh K, Mehra MR, Lavie CJ, Rizk Y, Forthal DN. Pharmaco-immunomodulatory therapy in COVID-19. Drugs. 2020;80:1267-92.

79. Sanchis-Gomar F, Lavie CJ, Perez-Quilis C, Henry BM, Lippi G. Angiotensin-converting enzyme 2 and antihypertensives (Angiotensin Receptor Blockers and Angiotensin-Converting Enzyme Inhibitors) in Coronavirus disease 2019. Mayo Clin Proc. 2020;95:1222-30. 